\title{
Analysis On Short-Term Change of Macular Function and The Correlates After Intravitreal Conbercept for CRVO-ME
}

\section{Yun-Chang Wang ( $\nabla$ wycocular@163.com )}

Heibei Eye Hospital, The key Laboratory of ocular of Heibei Province, The Treatment Centre of Ocular Disease of Hebei Province

\section{Rong-Rong Li}

Heibei Eye Hospital, The key Laboratory of ocular of Heibei Province, The Treatment Centre of Ocular Disease of Hebei Province

\section{Yi Cai}

Heibei Eye Hospital, The key Laboratory of ocular of Heibei Province, The Treatment Centre of Ocular Disease of Hebei Province

\section{Zi-Yi Wei}

Heibei Eye Hospital, The key Laboratory of ocular of Heibei Province, The Treatment Centre of Ocular Disease of Hebei Province

\section{Chang-Liang Shao}

Heibei Eye Hospital, The key Laboratory of ocular of Heibei Province, The Treatment Centre of Ocular Disease of Hebei Province

\section{Research Article}

Keywords: non-ischemia central retinal vein occlusion, macular edema, conbercept, optical coherence tomography, multifocal electroretinogram

Posted Date: October 11th, 2021

DOl: https://doi.org/10.21203/rs.3.rs-956113/v1

License: (c) (1) This work is licensed under a Creative Commons Attribution 4.0 International License. Read Full License

Version of Record: A version of this preprint was published at BMC Ophthalmology on April 8th, 2022. See the published version at https://doi.org/10.1186/s12886-022-02381-3. 


\section{Abstract}

-AIM: To study the short-term change of macular function and the correlates after intravitreal conbercept for CRVO-ME

•STUDY DESIGN: Prospective,clinical study.

-METHODS: 23 patients(23 eyes) were recruited, who were non-ischemia central retinal vein occlusion dianosed by FFA (fundus fluorescein angiography) and treated with intravitreal conbercept for macular edema. best - corrected visual acuity ( BCVA ),central macular thickness(CMT), amplitude density of P1 wave and implicit time of P1,N1 wave from ring 1 and ring 2 of mf-ERG were measured before and 1 week囚2month after treatment.

-RESULTS: Compared to the baseline, BCVACMTロamplitude density of P1 wave and implicit time of $\mathrm{P} 1, \mathrm{~N} 1$ wave from ring 1 and ring 2 were greatly improved at $1 \mathrm{~W} \otimes 2 \mathrm{M}$ after treatment; better results were gained at $2 \mathrm{M}$ compared to $1 \mathrm{~W}$; Pearson correlation analysis shows no significantly correlation between the improvement of mf-ERG with the change of BCVAロCMT.

-CONCLUSION: The BCVA】the strcture and the function of macular were greatly improved after intravitreal conbercept for central retinal vein occlusion induced macular edema; therefore no significantly correlation between the improvement of the function of macular with the strcture of macular and BCVA.

\section{Introduction}

Central retinal vein occlusion(CRVO) is a common disorder of retinal circulation, which is related to age, systemic diseases, retinal hemodynamic abnormalities, etc. It can be divided into the non-ischemic type and ischemic type according to the area and distribution of capillary non- perfusion zone. Macular edema is one of the main factors affecting the visual prognosis. Anti-VEGF has become the front-line treatment of CRVO-ME, ${ }^{[1]}$ which can reduce vascular permeability and reduce the absorption of fluid intra or subretina, thus the patients' BCVA improved.Optical coherence tomography (OCT) is used to measure the changes of fluid sub-retina of the macular and quantitatively evaluate the morphological recovery of the macular. Multi-focal electroretinography (MF-ERG) is a retinal function related to the activity of cone cells of the posterior under the state of light adaptation. It applied a pseudo-random binary m-sequence cycle to control, the small areas of the stimulation field are alternately overlapped to flash or graphic stimulation. As an objective examination, it can quantitatively analyze the electrical activity of the posterior pole at $30^{\circ}$, especially macular fovea cells. ${ }^{[2]}$ This paper discusses the changes of the macular function and its correlation with BCVA, CMT improvement within a short time after anti-VEGF, as well as investigates the toxicity of conbercept.

1 Objects and methods 
1.1 Participants: From August to November 2020, 23 patients (23 eyes) were diagnosed as CRVO-ME, including 10 males and 13 females, aged 52-71 years (average 62.48 \pm 5.50 ). Inclusion criteria: (1) All patients underwent complete examination including BCVA, intraocular pressure, slit-lamp biomicroscopy and fundus pre-set lens; (2) Non-ischemic central retinal vein occlusion diagnosed by FFA (Fundus fluorescein angiography) and macular edema diagnosed by OCT; (3) The enrolled patients voluntarily participated in this trial, signed informed consent and completed treatment and follow-up; (4) The intraocular pressure was in the normal range (10-21 $\mathrm{mmHg}$ ). Exclusion criteria: (1) Those with a previous history of intraocular surgery, retinal laser photocoagulation, intravitreal drug injection; Ischemic central retinal vein occlusion, age-related macular degeneration, diabetic retinopathy, idiopathic macular hole, epiretinal membrane, and other severe refracting opacity affected examinees. Patients and their families signed informed consent, and this study was approved by the Medical Ethics Committee of Hebei Eye Hospital.

\subsection{Methods}

1.2.1Procedures All patients enrolled were given a single intravitreal injection of conbercept. According to the requirements of routine intraocular surgery, after three times of anesthesia with $20 \mathrm{~g} / \mathrm{L}$ pramipecace hydrochloride eyedrops, the conjunctival sac was flushed with $50 \mathrm{~g} / \mathrm{L}$ povidone iodine and normal saline, The injections were performed in the operating theater with a sharp 30-gauge needle. The needle was inserted into the eye through the pars plana (3.5-4mm posterior from the limbus), $0.05 \mathrm{ml}$ of solution containing $0.5 \mathrm{mg}$ of conbercept was injected. After the operation, levofloxacin eyedrops were used four times /day for one week.

1.2.2 Measurement indexes patients were examined with BCVA, OCT, mf-ERG at baseline,one week and two months after the operation. The best corrected visual acuity was documented in Snellen VA, which was converted into logMAR VA for statistical analysis. The thickness of the fovea was measured by Spectralis OCT in Heidelberg, Germany. According to ISCEV ${ }^{[3]}$ (International Society for Clinical Electrophysiology of Vision) standard, Germany Roland RETI-Port/Scan 21 multifocal visual electrophysiology examination system was used. Sixty-one stimulation units were selected to stimulate the $30^{\circ}$ area of the posterior pole alternately and repeatedly, and the implict times of N1 and P1 in the first ring and the second ring and the amplitude density of the P1 wave were recorded. Follow-up was performed up to two months after the operation, complications such as abnormal intraocular pressure, endophthalmitis, vitreous hemorrhage recorded.

Statistical analysis: All statistical analyses were carried out using SPSS 23.0 (SPSS, Inc., Chicago, IL, USA), Quantitative data were presented as mean $\pm S D$. One-way ANOVA was used to compare the data,LSD- $t$ test was used to compare the two parametric data between each group, and Pearson correlation analysis was used to analyze the correlation between the improvement of P1 wave amplitude density and the changes of BCVA and CMT: $|\gamma|=0$, indicating that there is no correlation between the two variables, extremely weak correlation or no correlation for $0<|r|<0.2$, the weak correlation for $0.2<|r|<0.4$, 
the moderate correlation for $0.4<|r|<0.6$, the strong correlation for $0.6<|r|<0.8$, the extremely strong correlation for $0.8<|r|<1.0$. A $P$ value $<0.05$ was considered statistically significant.

2 Results All the indexes of the patients in this study were significantly improved at one week and two months after treatment $(P<0.05)$, which are presented in Table 1. The results showed that the macular morphology and function were significantly improved in a short time after anti-VEGF. Pearson correlation analysis: the correlation coefficient between the increase of amplitude density of P1 wave and BCVA change is $r=-0.079, P=0.721$, the correlation coefficient between the increase of amplitude density of $\mathrm{P} 1$ wave and the improvement of CMT is $r=0.282, P=0.192$. The results showed that there was no correlation between the improvement of the function in the fovea and within $10^{\circ}$ with the changes of BCVA and CMT.

Table 1 Comparison of parameters before and after treatment $\overline{\mathrm{x}} \pm s$

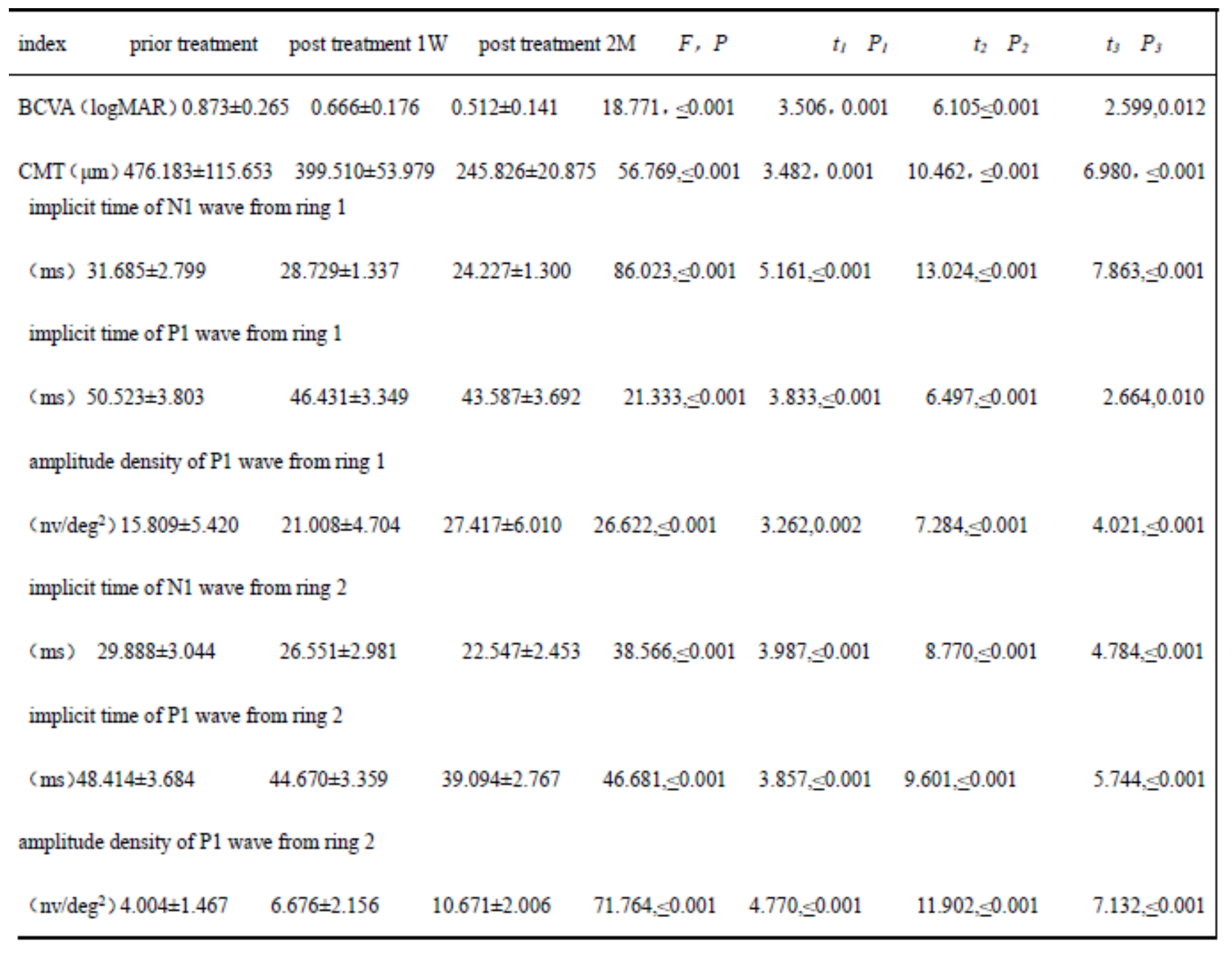

Note: $t_{1} P_{1}$ showed $1 \mathrm{~W}$ after treatment compared with baseline. $t_{2} P_{2}$ showed $2 \mathrm{M}$ after treatment compared with baseline. $t_{3} P_{3}$ showed $2 \mathrm{M}$ after treatment compared with $1 \mathrm{~W}$ after treatment.

\section{Discussion}


The abnormal hemodynamics and the destruction of the blood-retina barrier in CRVO patients lead to retinal edema due to fluid leakage, and the macular is more prone to fluid accumulation due to its loose tissue structure. With the expansion of the non-perfusion area, the release of VEGF increases, and the vascular permeability increases subsquently, macular edema represented. ${ }^{[4]}$ Long time edema can cause permanent damage, such as the loss of photoreceptor cells, the disorder of ellipsoid zone arrangement, the delay of P1 peak, and the decrease of amplitude, which are still irreversible after anti-VEGF treatment, which is related to the apoptosis caused by severe ischemia and hypoxia. With the application of optical coherence tomography angiography (OCTA) technology, the research on RVO-ME in recent years mainly focuses on the improvement of the macular structure and the re-perfusion of macular after anti-VEGF. ${ }^{[5-7]}$ The purpose of this study is to explore the functional change of foveal and the correlation after structure improvement.

The circulation of inner and middle layers is disturbed in CRVO patients. While mf-ERG originates from inner retinal cells and ON and OFF- bipolar cells. Tomoharu Nishimura ${ }^{[8]}$ confirmed that the accumulation of fluid will inhibit its function, subretinal fluid will also cause abnormal light transmission, and the sensitivity of cones to light will decrease, which will directly affect the transmission of synaptic signals between cones and bipolars.After intravitreal conbercept, the fluid will gradually decrease to absorption, and the function of cells will be partially restored, ${ }^{[9]}$ the implicit time and amplitude will gradually become normal, and mf-ERG will be significantly improved.However, after severe ischemic, axons of ganglion cells are broken and muller cells are irreversibly damaged.Even after absorption of fluid after anti-VEGF treatment, their electrical activity has not been improved, suggesting that macular function is permanently damaged.Nishijima et $a^{[10]}$ reported that VEGF, as a vascular factor, not only plays an important role in regulating the generation of vascular endothelial cells but also has a protective effect on the central nervous system and retinal ganglion cells. Animal experiments show that the decrease of intracellular VEGF concentration will significantly reduce ganglion cells. Under the condition of mild ischemia caused by CRVO, the $30-\mathrm{Hz}$ flicker response of full-field ERG will slightly increase. ${ }^{[11]}$ And the activity of cones is the main source of flicker response, the combination of VEGF and its receptors will activate inner retinal cells and cones, and the decreased level of VEGF in cells directly reduce the amplitude.The integrity of photoreceptor cells will be destroyed in macular edema. After anti-VEGF treatment for 3 months, the integrity of photoreceptor cells in some patients tends to be normal, and the visual recovery is positively correlated with the morphology of baseline photoreceptor cells. ${ }^{[12]} \mathrm{U}$ MIT U Beyt I ' Nan ${ }^{[13]}$ studied the ultrastructure of photoreceptors in the fovea of CRVO animal model by scanning an electron microscope. After intravitreal injection of bevacizumab, a series of changes occurred in mitochondria of photoreceptor inner segment cells, such as swelling, disordered arrangement, decreased density, loss of cristae, increase of amorphous corpuscles rich in phospholipids, and then apoptosis started.

Mitzy E.T. ${ }^{2]}$ reported that the functions of retinal ganglion cells and cone cells did not change in a short time after bevacizumab was used in AMD, high myopia CNV, PDR, and RVO.Rohit Shetty ${ }^{[14]}$ came to a different conclusion, research showed that $\mathrm{mf}$-ERG increased slightly to moderately in a short time after 
intravitreal injection of bevacizumab, but it was not directly related to the best corrected visual acuity and the improvement of retinal thickness in fovea maculae.Compared with monoclonal antibody drugs such as ranibizumab.Conbercept, however as a fusion protein, can bind to the extracellular domain 2 of VEGF receptor 1 and the extracellular domains 3 and 4 of VEGF receptor $2 .{ }^{[15]}$ This study showed that conbercept had the same or even better effect in RVO-ME patients. The conclusion of this study was consistent with Rohit Shetty's, after treatment, the macular function was significantly improved at one week and two months compared with the baseline, which confirmed that conbercept had no toxic effect on cones and inner retinal cells in a short time.The first and second rings of mf-ERG reflects the fovea and inner retinal function within $10^{\circ}$. The improvement of amplitude density and implicit time of P1 wave and N1 wave indicated that anti-VEGF could alleviate the ischemia and hypoxia condition of inner retina in a short time and break the positive feedback process of VEGF production.In this study, most patients suffered from photoreceptor cell loss and ellipsoid zone disorder before the treatment, and some patients had obvious changes in cell integrity and distribution two months after treatment, and the BCVA also improved significantly.

This study showed that the morphology and function of the macular of CRVO-ME patients improved significantly in a short time after anti-VEGF treatment and did not show the cytotoxic effect of anti-VEGF drugs, which was consistent with previous reports, but there was no correlation between them. There were several reasons for this: 1 . The subretinal fluid of the foveal was decreased after anti-VEGF treatment, and the central visual acuity was improved. Considering economic factors and the patient's ischemic state is not very serious, the intensity of anti-VEGF treatment was not enough, which made the functions of cones and inner retinal cells not fully recovered, so there was no correlation between the improvement of macular function and structure.2. Whether some patients had changed to ischemic central retinal vein occlusion within two months of follow-up; Also, because some patients are older, whether there were degenerative changes in macula during treatment and follow-up.3. Choriocaphillaris is the main source of blood supply to the macula, and its endothelial cells have loose junction, so oxygen is easy to diffuse to the inner layer cells. Whether conbercept would affect the permeability of its endothelial cells and reduce the intercellular space, thus reducing the fovea blood supply, have been rarely reported in the past.4. Because of the small number of samples, short follow-up time, and the lack of preoperative evaluation of choroidal blood supply, the size, and the location of retinal capillary non-perfusion area, etc, whether these factors will affect the experimental results remains to be demonstrated in the next step.Full-field ERG reflects the electrical activity of the whole retinal cells. The non-perfusion area of CRVO patients starts from the peripheral retina where a large number of rods are located. Whether its response to ischemic state is more sensitive than that of macular area, whether it has been damaged before the macular changes, whether the amacrine cells change reversibly after improvement of ischemia, whether circulation changes of the peripheral retinal synchronize with the macular area, and whether their changes are consistent and related, which need to be clarified by full-field ERG examination. We will focus on it in the follow-up study. 
To sum up, the BCVA, the structure, and the function of the macular were greatly improved after intravitreal conbercept for central retinal vein occlusion induced macular edema; therefore there was no significant correlation between the improvement of the function of macular with the structure of macular and vision.

\section{Declarations}

Ethics approval and consent to participate

This study was performed in accordance with the principles of the Declaration of

Helsinki, and was approved by the Institutional Review Board of Heibei Eye Hospital. All of the subjects signed informed consent forms.

Disclosure Statement

The datasets used and analyzed during the current study are available from the corresponding author on reasonable request囚The authors declare that they have no competing interests.

Consent for publication

there are no details on individuals reported within the manuscript, consent for publication of images are not be required.

Author Contributions

Rong-Rong Li conceived and designed the experiments; Yun-Chang Wang wrote the manuscript;

Yi Cai perform the data analyses; Zi-Yi Wei collect data and information; Chang-Liang Shao helped perform the analysis and follow up patients.

\section{References}

1. Sun, Z., Zhou, et al. Efficacy and safety of intravitreal conbercept injections in macular edema secondary to retinal vein cclusion. Retina 2017; 37:1723-1730

2. MITZY E. T, VICTORIA C,GERARDO G, et al. MULTIFOCAL ELECTROPHYSIOLOGIC FINDINGS AFTER INTRAVITREAL BEVACIZUMAB (AVASTIN) TREATMENT. Retina 2012; 32:972-976

3. Hood DC, Bach M, Brigell M, et al. International Society for Clinical Electrophysiology of Vision (2012) ISCEV standard for clinical multifocal electroretinography (mfERG) (2011 edition). Doc Ophthalmol 2012;124(1): 1-13

4. Yan Zhipeng, Wang Chenghu. Short-term efficacy of appercept in the treatment of macular edema secondary to retinal vein occlusion. International Journal of Ophthalmology 2020;20(7):1253-1256 
5. Yang Jing, Zhang YL, Yang Na, et al. Clinical efficacy and prognostic factors of ranibizumab in BRVO-ME. International Journal of Ophthalmology 2020;20(4):673-679

6. Li Jin, Zhang Shengjuan, Yan pei, et al. Effects of conbercept on retinal vascular density in macular area in patients with RVO secondary macular edema. International Journal of Ophthalmology 2019;19(9):1605-1608

7. Yan Zhipeng, Yao Jin.Macular vascular density in patients with non-ischemic central retinal vein occlusion.Recent Advances in Ophthalmology 2016;36(12):1165-1167

8. Tomoharu N, Shigeki Machida, Yuji Hara.Changes of focal macular and full-field electroretinograms after intravitreal aflibercept in patients with central retinal vein occlusion. Doc Ophthalmol 2020;10:1412(2)

9. SIVAKAMI A. PAI, ROHIT SHETTY, PRIYA B. VIJAYAN, et al.Clinical, Anatomic, and Electrophysiologic Evaluation Following Intravitreal Bevacizumab for Macular Edema in Retinal Vein Occlusion. Am J Ophthalmol 2007;143:601-606

10. Nishijima K, Ng YS, Zhong L, et al. Vascular endothelial growth factor-A is a survival factor for retinal neurons and a critical neuroprotectant during the adaptive response to ischemic injury. Am J Pathol 2007;171:53-67

11. Ryohei M, Mineo K, Kumiko K, et al. Supernormal Flicker ERGs in Eyes With Central Retinal Vein Occlusion: Clinical Characteristics, Prognosis, and Effects of Anti-VEGF Agent. Invest Ophthalmol Vis Sci.2018;59:5854-5861

12. Asaf A, Ayana K, Vitaly M, et al. Photoreceptor Integrity Predicts Response to Anti-VEGF Treatment.Ophthalmic Res 2017;571(1)

13. U"mit U" beyt I,Berrin A, Tuncay K,et al. Preclinical Safety Evaluation of Intravitreal Injection of FullLength Humanized Vascular Endothelial Growth Factor Antibody in Rabbit Eyes. Invest Ophthalmol Vis Sci.2007:48:1773-1781

14. Rohit S,Sivakami A. P,Ajoy V,et al. Electrophysiological and structural assessment of the central retina following intravitreal injection of bevacizumab for treatment of macular edema. Doc Ophthalmol.2008:116:129-135

15. X. Chen, T. M. Hu, J. Zuo, et al.Intravitreal conbercept for branch retinal vein occlusion induced macular edema:one initial injection versus three monthly injections. BMC Ophthalmology 2020:20:225-232

\section{Figures}




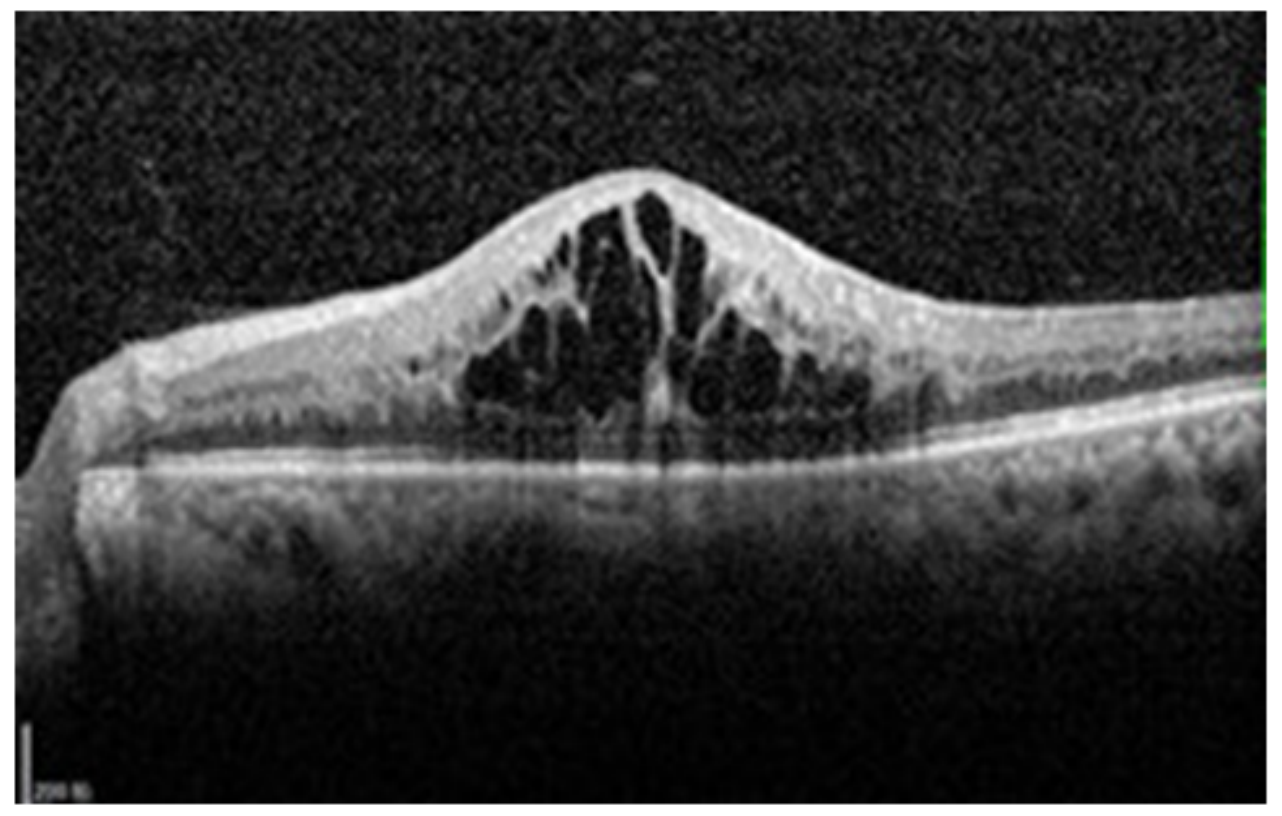

\section{Figure 1}

Baseline multiple low refections of cystoid can be seen in fovea,particle-like strong reflection signal can be seen intra-retina, the ellipsoid zone was broken with continuity

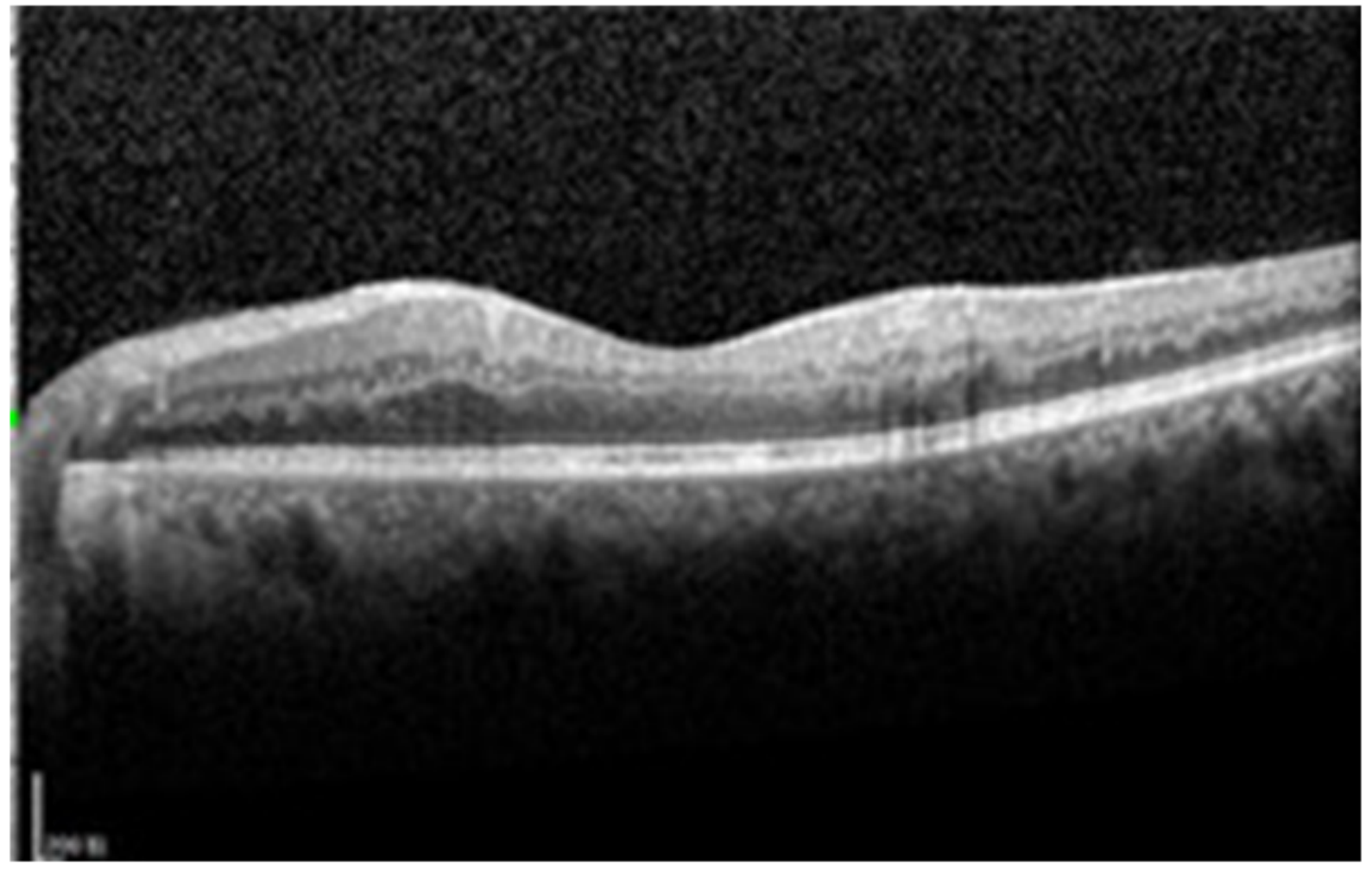

\section{Figure 2}

The same patient two months after treatment, the fovea is close to normal 

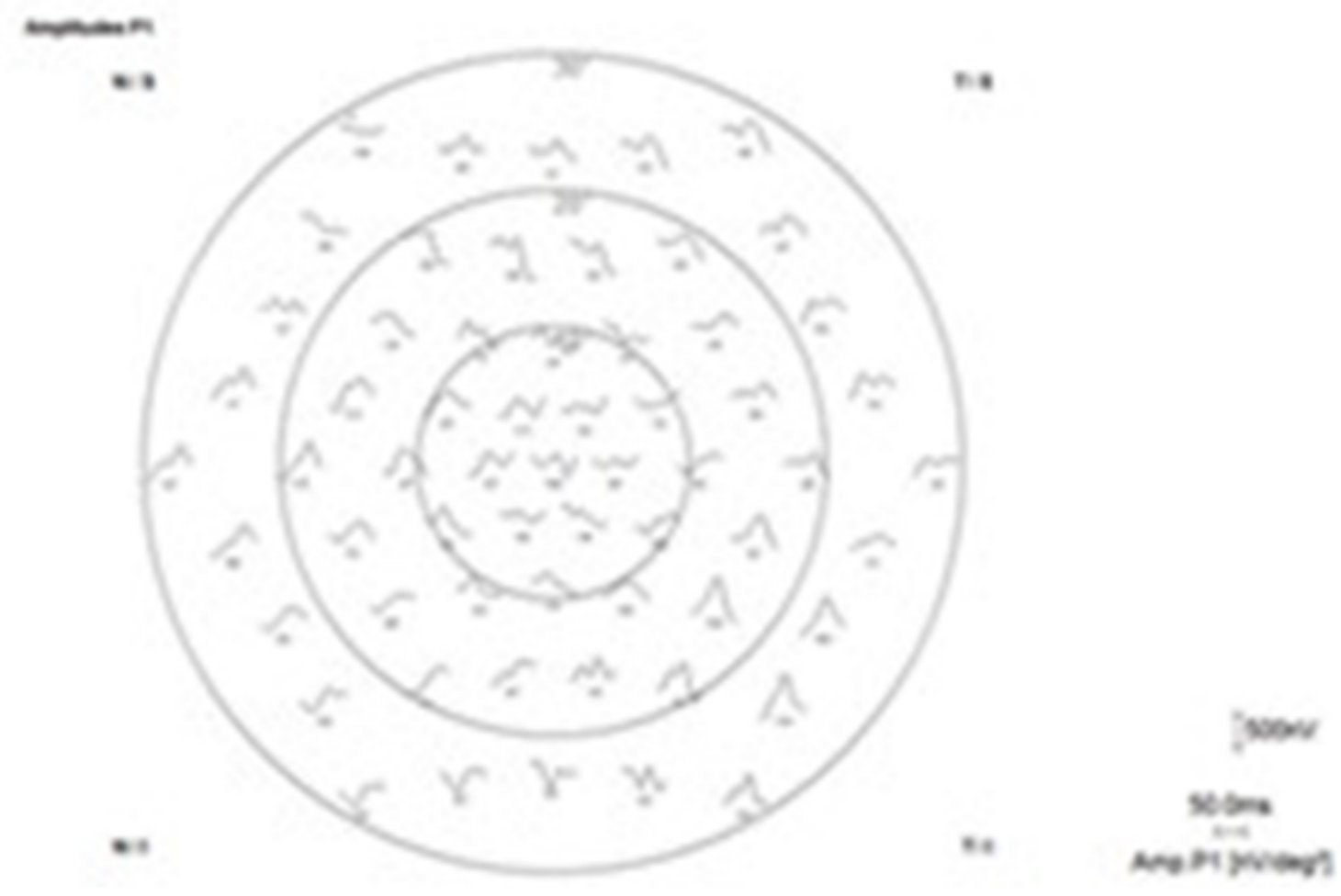

Figure 3

Plots image of a patient before the treatment
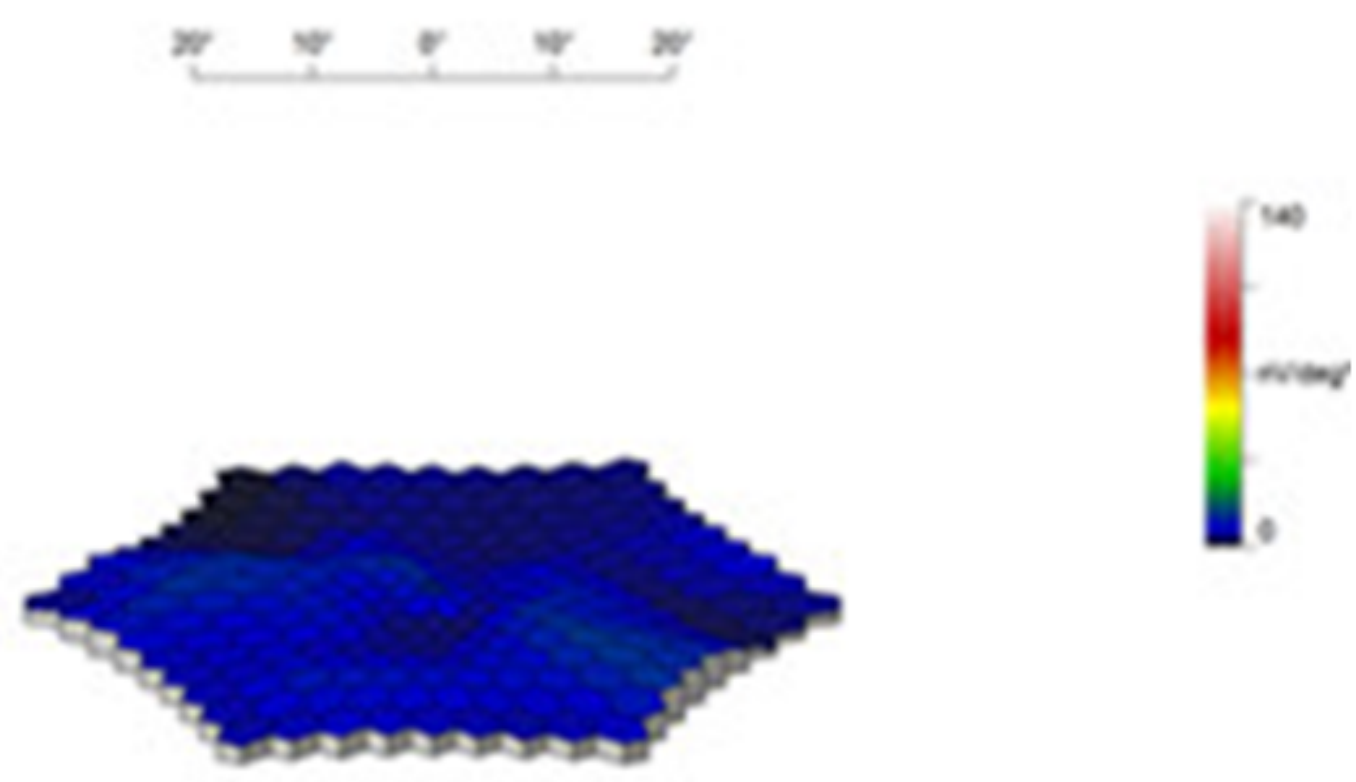

Figure 4

$3 \mathrm{D}$ image of the same patient before the treatment 

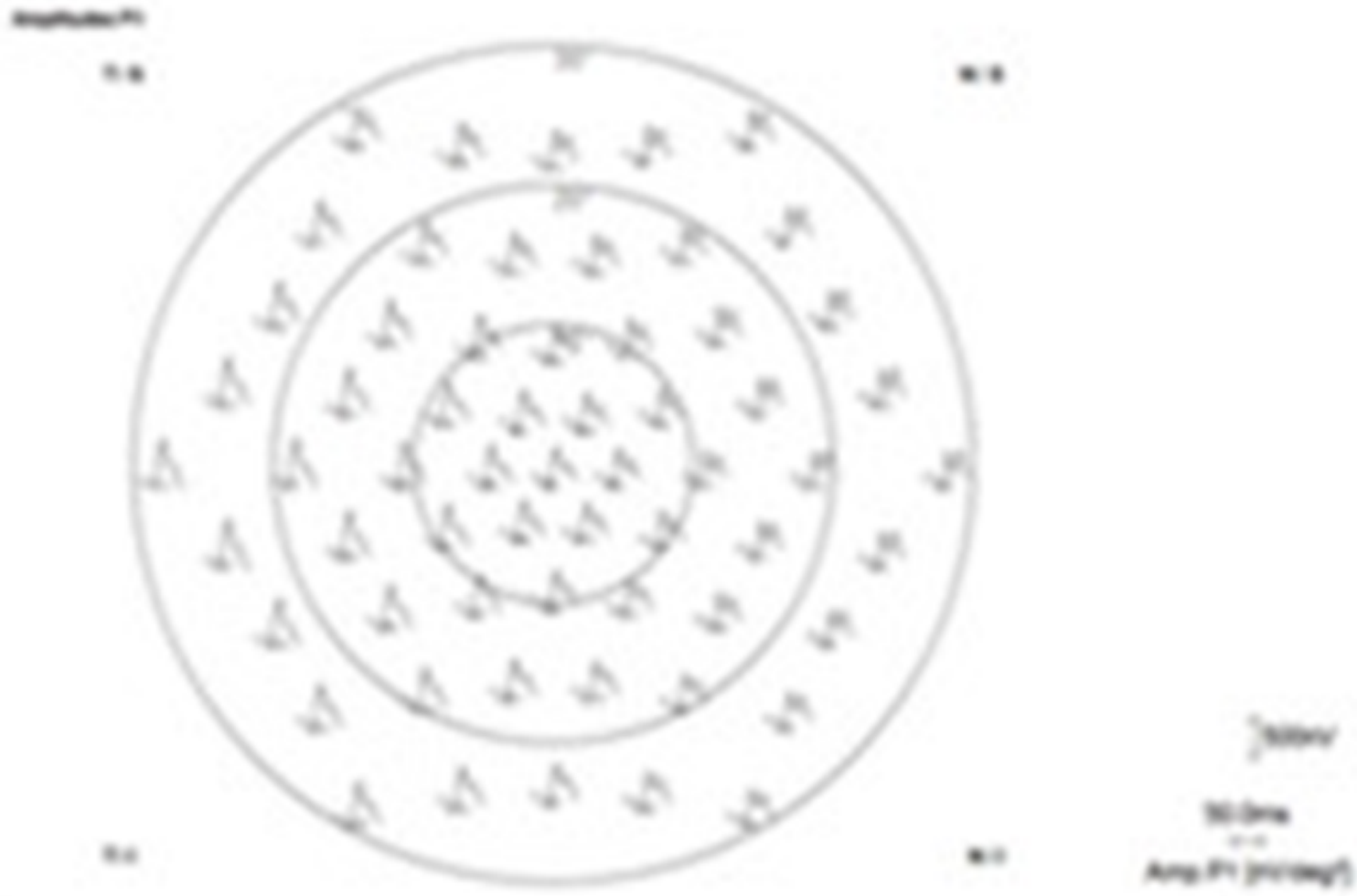

Figure 5

Plots image of the same patient two months after the treatment
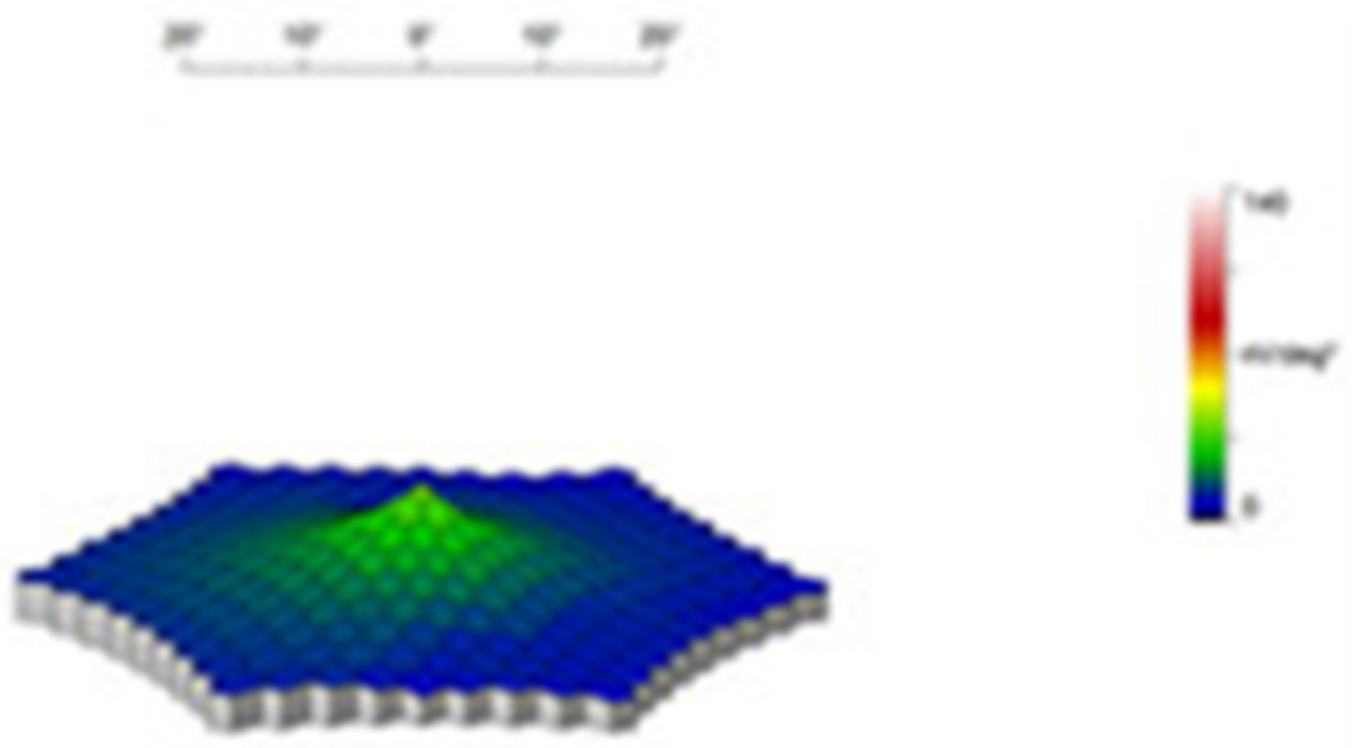

Figure 6 
$3 \mathrm{D}$ image of the same patient two months after the treatment 